\title{
Nucleation and Growth of Metal Films and Nanocrystals on Two-dimensional Materials
}

\author{
Joachim Dahl Thomsen, Kate Reidy, Thang Pham and Frances Ross \\ Massachusetts Institute of Technology, Cambridge, Massachusetts, United States
}

Beyond graphene, many two-dimensional materials (2DMs) have now been isolated with an exciting range of properties: insulators, semiconductors, metals, semimetals, superconductors, topological insulators, piezoresistors, and ferromagnets. More than 1800 of these 2DMs have been predicted to exist [1]. However, the full potential of this diverse set of materials can only be fully exploited if 2DMs can be interfaced in a well-controlled fashion with the common 3DMs used in technology, such as metals or semiconductors, either as nanoscale crystals or larger area contacts. The structure and defects at the 2D/3D interface influence properties such as contact resistance, photoresponse and high frequency performance [2]. Thus, a fundamental understanding of interfacial phenomena such as crystallographic alignment, defects, strain and growth mode is important for the continued and rapid development of 2D material devices.

Here we study the nucleation and growth of a variety of metals, including $\mathrm{Ag}, \mathrm{Au}, \mathrm{Ti}$ and $\mathrm{Cr}$, commonly used in electronics, and $\mathrm{Nb}$, a superconductor, on different 2DMs (graphene ( $\mathrm{Gr}$ ), hexagonal boron nitride $(\mathrm{hBN}), \mathrm{WSe}, \mathrm{MoS}_{2}$ ) and 2DM heterostructures. We use transmission electron microscopy (TEM) to provide direct insight into crystal size, shape and orientation, epitaxy, and diffusivity via nucleation density. We first transfer 2DMs to TEM sample carriers that allow in-situ Joule heating in ultrahigh vacuum (UHV) conditions and subsequently evaporate metals onto the 2DM without breaking vacuum. Annealing and evaporation in the same system is necessary to remove contamination arising from the polymer handle used for the transfer and to avoid contamination from the environment, both of which will provide heterogeneous nucleation sites that we find lead to polycrystalline and non-epitaxial metal nanocrystals. The TEM sample carriers are home-made silicon chips with a free-standing silicon nitride membrane etched with $4 \mu \mathrm{m}$ diameter holes. Besides the basic parameters that affect growth mode and epitaxy such as diffusivity, binding and cohesive energies [3], we explore the effects of 2DM thickness, 2DM temperature during metal deposition, and the material (substrate) beneath the 2DM ( $\mathrm{SiO}_{2}, \mathrm{hBN}$, or no substrate/suspended). We investigate different substrates by depositing the metals onto 2DMs placed on these substrates and then transfer the 2DMs to TEM sample carriers for imaging.

Temperature during deposition is expected to affect the diffusivity of metal atoms on the 2D surface. We show for $\mathrm{Au}$ and $\mathrm{Nb}$ on $\mathrm{Gr}$ that, as expected from crystal growth models, temperature determines whether the crystal morphology is dendritic or compact and faceted. Fig. 1(a,b) shows Au deposited on $\mathrm{Gr}$ at $\sim 50$ ${ }^{\circ} \mathrm{C}$ and $100{ }^{\circ} \mathrm{C}$, respectively. Fig. 1(c, d) shows $\mathrm{Nb}$ deposited on $\mathrm{Gr}$ at room temperature and $900{ }^{\circ} \mathrm{C}$, respectively. In both cases, higher temperature produces more compact and faceted islands with improved epitaxial alignment. Au is face centered cubic with orientations $\mathrm{Au}(111) \| \operatorname{Gr}(0001)$ and $\mathrm{Au}[220] \| \mathrm{Gr}[10-$ 10], while $\mathrm{Nb}$ is body centered cubic with $\mathrm{Nb}(111) \| \mathrm{Gr}(0001)$ and $\mathrm{Nb}[110] \| \mathrm{Gr}[11-20]$ for the strongest $\mathrm{Nb}[110]$ peak, accounting for about $80 \%$ of the spot intensity, while the remainder is rotated $30^{\circ}$ so that $\mathrm{Nb}[110] \| \mathrm{Gr}[11-20]$.

The effect of the substrate beneath the 2DM on the epitaxy and crystal morphology is relatively unexplored, but we find that the substrate greatly influences these parameters. Fig. 2(a) shows Au on hBN that was suspended during deposition. The morphology is similar to Au on Gr, with compact triangular 
crystals, see blue inset in Fig 2(a). However, polymer residue on these samples nucleates non-epitaxially aligned crystals (red inset). In contrast, Fig. 2(b) shows Au deposited on $20 \mathrm{~nm}$ thick hBN supported on $\mathrm{SiO}_{2}$ that was subsequently transferred to a TEM sample carrier. The $\mathrm{Au}$ is not faceted, and a higher nucleation density is seen. A similar effect is seen for graphite on $\mathrm{SiO}_{2}$. Due to the relatively large distance between the $\mathrm{SiO}_{2}$ and the top surface of the 2DM, this suggests that substrate effects like surface charges play little role in the crystal growth. Rather, 2DM roughness may be a determining parameter [4]. Roughness is known to be lower for suspended 2DMs [5], suggesting a longer diffusion distance and hence fewer but larger nanocrystals.

In suspended layers we find that the 2DM thickness is important: nucleation density and hence diffusion distance depend on 2DM thickness. We obtain the longest diffusion distances, $>2 \mu \mathrm{m}$, on suspended fewlayer $\mathrm{Gr}>8$ monolayers thick. Because the sites for spontaneous nucleation can be very far apart, it is possible to purposely create nucleation sites with a smaller separation using helium ion microscopy. This defect engineering may be useful for tunable plasmonic resonator arrays for biosensing or photodetection.

The results presented here may impact the design of future devices. The key question is whether the different metal morphologies that we have seen will affect the physical properties of the final devices. As an example, the contact resistance between metals and semiconducting 2DMs must be reduced beyond current state-of-the-art in order to take advantage of the electrical properties of the 2D materials [2,6]. Our sample design and fabrication scheme allow us to correlate contact resistance with the structure of the metal-2DM interface directly, providing input into strategies for device fabrication.
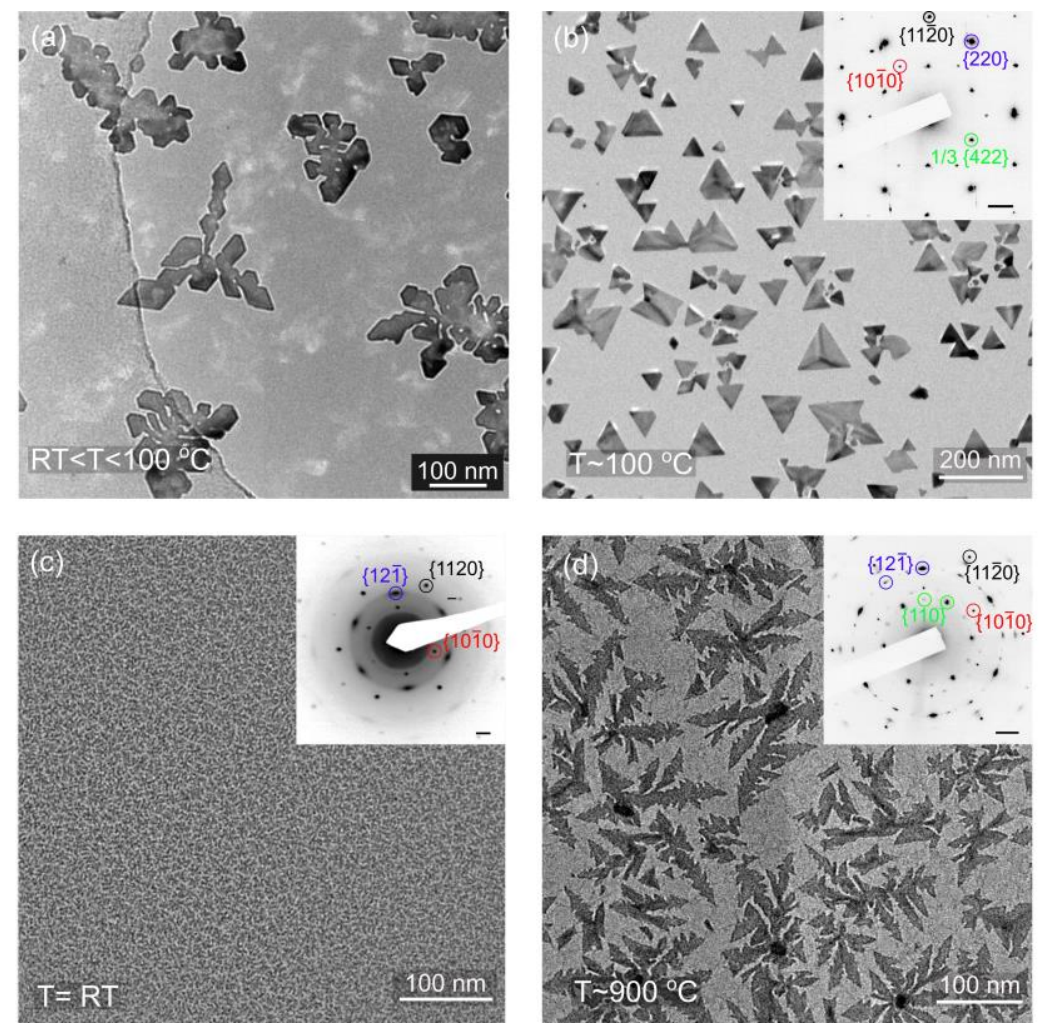

Figure 1. TEM images showing the effect of temperature during metal deposition on the metal nanocrystal morphology. (a, b) Au deposited on graphene at a temperature of around $50{ }^{\circ} \mathrm{C}$ and $100{ }^{\circ} \mathrm{C}$, respectively. 
(c, d) $\mathrm{Nb}$ deposited on graphene at room temperature and $900{ }^{\circ} \mathrm{C}$, respectively. Insets in (b, c, d) show diffraction patterns of the approximate regions in the main images, with scale bar $2 \mathrm{~nm}^{-1}$.
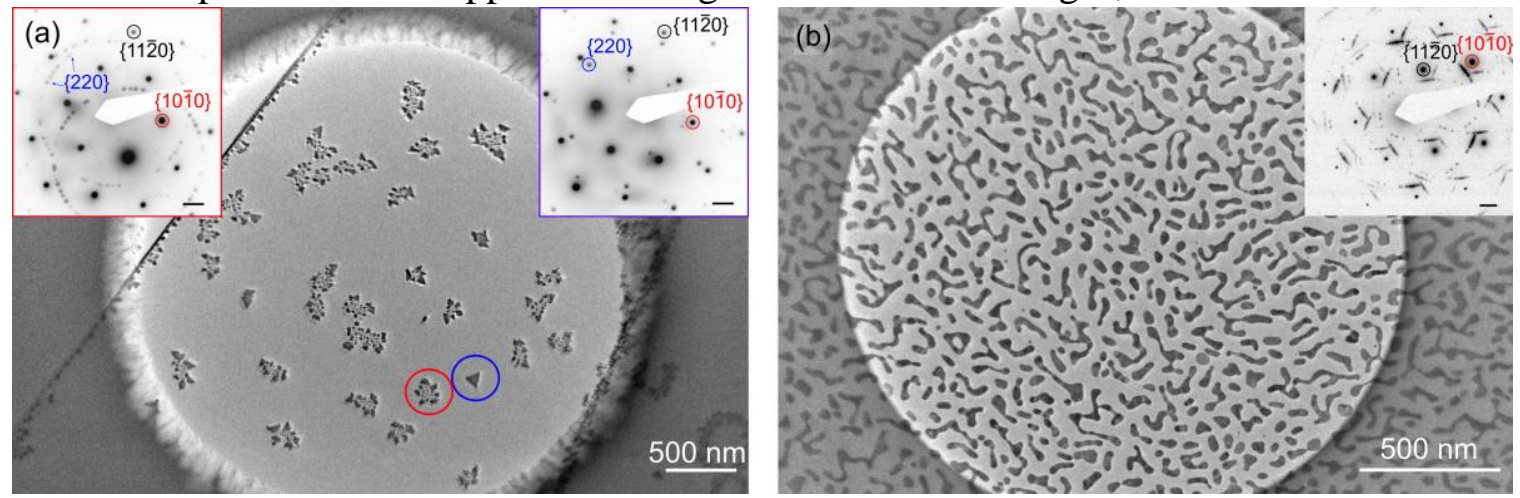

Figure 2. TEM images showing the effect of substrate on Au morphology. (a) Au deposited on suspended $\mathrm{hBN}$. The blue and red circles indicate the area from which the selected area diffraction patterns in the blue and red boxes, respectively, are acquired. The blue circle marks a triangle which has nucleated alone. (b) Au deposited on hBN while the $\mathrm{hBN}$ was supported on a $\mathrm{Si} / \mathrm{SiO}_{2}$ substrate and subsequently transferred to a TEM sample carrier for imaging. Insets: diffraction patterns of the samples, scale bar $2 \mathrm{~nm}^{-1}$.

\section{References}

[1] N. Mounet et al., "Two-dimensional materials from high-throughput computational exfoliation of experimentally known compounds", Nat. Nanotech., 13, 246-252 (2018)

[2] A. Allain et al., "Electrical contacts to two-dimensional semiconductors", Nat. Mat., 14, 1195-1205 (2015)

[3] C. Gong et al., "Metal contacts on physical vapor deposited monolayer MoS2", ACS Nano, 7(12), 11350-11357 (2013)

[4] L. Liu et al., "Slow gold adatom diffusion on graphene: effect of silicon dioxide and hexagonal boron nitride substrates", J. Phys. Chem. B, 117, 4305-4312 (2013)

[5] J. D. Thomsen et al., "Suppression of intrinsic roughness in encapsulated graphene", PRB, 96, 014101 (2017)

[6] Y. Xu et al., "Contacts between Two- and Three-Dimensional Materials: Ohmic, Schottky, and p-n Heterojunctions”, ACS Nano, 10, 4895-4919 (2016) 